\title{
MedienPädagogik
}

Zeitschrift für Theorie und Praxis der Medienbildung

\section{Lernen durch Videos - Empirische Befunde zur Gestaltung von Erklärvideos}

\author{
Stefanie Findeisen, Sebastian Horn und Jürgen Seifried
}

\begin{abstract}
Zusammenfassung
Sowohl im privaten Umfeld als auch im schulischen Bereich gewinnen Erklärvideos zunehmend an Bedeutung. Empirische Befunde belegen positive Effekte von Erklärvideos für kognitive (Wissenszuwachs) sowie für nicht-kognitive Faktoren (z.B. Motivation, Aufmerksamkeit). Dies gilt sowohl für die Rezeption als auch die Produktion von Videoerklärungen. Darüber hinaus befassen sich empirische Studien zunehmend mit der Analyse der Wirkung verschiedener Gestaltungselemente von Erklärvideos (z.B. Interaktivität, Eigenschaften der erklärenden Person). Der Beitrag verfolgt das Ziel, vorliegende empirische Befunde zu verschiedenen Gestaltungselementen zu systematisieren. Zu diesem Zweck wurden 24 Studien gesichtet, die sich mit der Wirkung einzelner Gestaltungselemente befassen. Auf Basis der Befunde werden Gestaltungsempfehlungen für Erklärvideos ausgesprochen. Es zeigt sich, dass insbesondere der Einsatz interaktiver Elemente bedeutsam für den Lernerfolg ist. Zudem wirken sich die gewählte Perspektive (d.h. die Entscheidung, ob das Video aus der Sicht des Erklärenden oder der Person, die das Modell beobachtet, gezeigt wird) sowie das Design (z.B. Ästhetik) signifikant auf den Lernerfolg aus. Mit Blick auf die erklärende Person scheint lediglich das Alter bedeutsam zu sein - nicht jedoch das Geschlecht oder deren Sichtbarkeit im Erklärvideo.
\end{abstract}

Learning through videos - Empirical findings on the design of explanatory videos

\begin{abstract}
Explanatory videos are becoming increasingly important in both private learning efforts and instruction in school. Empirical findings suggest positive effects of explanatory videos on learning success as well as on non-cognitive factors (e.g. motivation, engagement). This applies to both the reception and the production of video explanations. In addition, empirical studies are increasingly concerned with the investigation of various design elements of explanatory videos (e.g. interactivity, characteristics of the instructor). The aim of this article is to systematize existing empirical findings on various design elements. For this purpose, 24 studies that deal with the effects of individual design elements will be reviewed. Based on these findings, design recommendations for explanatory videos are derived. It is shown that in particular the use of interactive elements is important for learning success. In addition, the video perspective (the decision whether to show the video
\end{abstract}


from the perspective of the person explaining or the person observing the model) as well as the design (e.g. aesthetics) have a significant effect on learning success. With regard to the instructor, only the age seems to be significant - not the gender or the visibility in the explanatory video.

\section{Erklären durch Videos - Forschungsfeld und -ansätze}

Immer mehr Jugendliche nutzen YouTube oder andere Videoportale für schulische Zwecke, z.B. um sich eigenständig Lerninhalte anzueignen oder nachzuarbeiten (Wolf 2015). Studien verweisen darauf, dass sich $45 \%$ aller befragten Internetnutzer ab 14 Jahren schon einmal ein Video-Tutorial angesehen haben (Bitkom 2017). Über ein Fünftel der befragten Jugendlichen nutzen regelmässig Videotutorials zu ausserschulischen Themen, und für schulische Themen beträgt der Wert immerhin noch $13 \%$ (Medienpädagogischer Forschungsverbund Südwest 2017). Bei einer Befragung von rund 250 Schülerinnen und Schülern gaben knapp zwei Drittel der Befragten an, Videos zur Vorbereitung von Klausuren, Referaten und Präsentationen zu verwenden (Rummler und Wolf 2012). Diese Zahlen geben einen ersten Eindruck von der Bedeutung (zumindest aus Sicht der Lernenden) und Verbreitung von entsprechenden Formaten.

Darüber hinaus deuten empirische Befunde darauf hin, dass sich die Rezeption von Erklärvideos positiv auf die Lernleistung auswirkt. In einschlägigen Studien wird beispielsweise gezeigt, dass Lernende nach der Nutzung von Erklärvideos über höheres prozedurales Wissen (Bedienen einer Software) verfügen als Lernende, welche sich die Inhalte anhand papierbasierter Unterlagen erarbeiteten ( $\mathrm{H}$. van der Meij und J. van der Meij 2014; Lloyd und Robertson 2012). Zudem beeinflussen Erklärvideos Aufmerksamkeit, Bedeutsamkeitsempfinden und Engagement der Lernenden positiv (Hartsell und Yuen 2006). Lernen durch den Konsum von Videos ist allerdings nur eine Seite der Medaille. Auch der eigenständigen Produktion von Erklärvideos wird Lernpotenzial zugeschrieben, da diese Schülerinnen und Schülern eine intensive Auseinandersetzung mit der darzustellenden Thematik und deren didaktische Aufbereitung abverlangt (Schön 2013). Empirische Studien verweisen auf positive Effekte durch das (gemeinschaftliche) Erstellen von Videoerklärungen sowohl für den Lernerfolg als beispielsweise auch für Zufriedenheit, Motivation oder Autonomieerleben der Lernenden (Slopinski 2016; Spires et al. 2012; Zahn et al. 2010; Fiorella und Mayer 2013, 2014; Hoogerheide, Loyens und van Gog 2014b).

Ungeachtet der Vielzahl an Studien zur Wirkung von Erklärvideos fehlt es jedoch nach wie vor an didaktischen und formalen Qualitätskriterien entsprechender Formate (Schön 2013). Daher verfolgt der vorliegende Beitrag das Ziel, auf Basis empirischer Befunde bedeutsame Merkmale für Erklärvideos herauszuarbeiten und entsprechende Gestaltungsempfehlungen abzuleiten. Der Fokus liegt dabei auf der 
Videonutzung (Rezeption) in formalen Bildungskontexten. Im Folgenden gehen wir zunächst auf zentrale Charakteristika und verschiedene Formen von Erklärvideos ein und thematisieren lehr-lern-theoretische Überlegungen. Im Anschluss wird ein Überblick über einschlägige empirische Befunde zur Wirkung verschiedener Gestaltungselemente von Erklärvideos gegeben. Abschliessend werden auf Basis der aufgezeigten Effekte Qualitätskriterien für die Gestaltung von Erklärvideos herausgearbeitet.

\section{Begriffsabgrenzung und Charakterisierung von Erklärvideos}

Erklärungen sind zentraler Bestandteil des Unterrichts (Leinhardt 2010; Pauli 2015; Ball, Hill und Bass 2005). Ein Erklärprozess ist grundsätzlich durch drei zentrale Merkmale charakterisiert (Findeisen 2017). Erklären findet (1) als Interaktion zwischen einem Erklärenden und mindestens einem Zuhörenden statt. (2) Der Erklärende verfügt gegenüber dem/n Zuhörenden über einen Wissensvorsprung (Wissensasymmetrie); die Erklärung thematisiert also Inhalte, die dem/n Zuhörenden zunächst nicht bekannt bzw. bewusst sind. (3) Der Erklärende verfolgt die Zielsetzung, dem Gegenüber bestimmte Inhalte verständlich zu machen. Beim Erklären geht es also nicht um die Präsentation von Fachinhalten, sondern um deren Verständlichkeit. Verstehen ist damit gleichzeitig das Mass für den Erfolg sowie für die Qualität einer Erklärung.

Mit Blick auf die vielfältigen Formen von Erklärvideos ist für die folgenden Ausführungen eine begriffliche Abgrenzung dienlich. In Anlehnung an Wolf (2015) werden im vorliegenden Beitrag Video-Tutorials als Subgenre von Erklärvideos verstanden, und es wird folgende Definition zu Grunde gelegt:

Erklärvideos sind eigenproduzierte, kurze Filme, in denen Inhalte, Konzepte und Zusammenhänge erklärt werden (Erklärvideos im engeren Sinne) oder Tätigkeiten und Prozesse demonstriert und kommentiert werden (Tutorial), jeweils mit der Intention, beim Betrachter ein Verständnis zu erreichen bzw. einen Lernprozess auszulösen.

Die hier gewählte Begriffsauffassung verweist auf ein Mindestmass an Didaktisierung. Erklärvideos unterscheiden sich somit auf der einen Seite von Dokumentarfilmen, welche teilweise einen geringen Grad an Didaktisierung aufweisen, vor allem dann, wenn sie vornehmlich informieren und Fakten ohne weiterführende Erklärung präsentieren. Auf der anderen Seite sind Erklärvideos von professionell produzierten Lehrfilmen zu unterscheiden, die einen hohen Grad an Didaktisierung aufweisen und den Anspruch auf Vollständigkeit und Fehlerfreiheit erheben. Erklärvideos sind in der Regel eher kurz (maximal 20 Minuten; Schaarschmidt, Albrecht und Börner 2016) und beziehen sich auf einzelne Themenausschnitte. Daher erheben Erklärvideos keinen Anspruch auf Vollständigkeit - insbesondere dann nicht, wenn diese durch Schülerinnen und Schüler erstellt wurden (Lübcke und Burchert 2013; Wolf 2015). Die skizzierte Kategorisierung ist indes nicht trennscharf. Eigenproduzierte 
Videoblogs, in denen persönliche Erfahrungen und Tipps geteilt werden, bieten eine Art Lebenshilfe (z.B. Umgang mit einem bestimmten Problem). Hierbei finden sich zwar teilweise erklärende Elemente, grundsätzlich ist der Grad der Didaktisierung aber gering und die Intention muss nicht im Erklären oder in der Wissensvermittlung liegen. Zum anderen kann die Unterscheidung zwischen Video-Tutorials und Performanzvideos (letztere zeigen eine beobachtbare Fertigkeit ohne weitere didaktische Aufarbeitung) verschwimmen, wenn letztere um Gestaltungselemente ergänzt werden, die das Nachmachen fördern sollen (Wolf 2015). ${ }^{1}$

\section{Lernen am Modell}

Das Lernpotenzial von Videos kann mit Rückgriff auf die sozialkognitive Lerntheorie von Albert Bandura $(1977,1986)$ bestimmt werden. Bandura postulierte, dass Beobachterinnen und Beobachter ein kognitives Schema des Verhaltens eines Modells erwerben, welches es innen erlaubt, das beobachtete Verhalten zukünftig selbst auszuführen. Der Kompetenzerwerb durch Lernen am Modell setzt Aufmerksamkeit und Gedächtnisprozesse während des Betrachtens des Modells voraus. Für eine erfolgreiche Ausführung der Tätigkeit (Performanz) sind sowohl kognitive Fähigkeiten als auch motivationale Aspekte hilfreich (Schnotz 2011). Folgt man der obenstehenden Definition, so sind insbesondere Video-Tutorials (vollständige Handlung, explizite Aufforderung zum Nachahmen, höherer Grad an Didaktisierung; Wolf 2015) geeignet für Modelllernen. Wie gut das Nachahmen des Modells gelingt, hängt von verschiedenen Faktoren ab, u.a. von der Komplexität und Sichtbarkeit der Handlung oder dem Vorwissen der Betrachtenden (Rummler 2017). Zudem sind einzelne Gestaltungselemente des Videos von Bedeutung (siehe hierzu die folgenden Abschnitte).

\section{Unterschiede zwischen Erklärvideos und Unterrichtserklärungen}

Während Unterrichtserklärungen typischerweise entweder mündlich oder schriftlich erfolgen (Leinhardt 2001; Wittwer und Renkl 2008), sind Videoerklärungen charakterisiert durch Multimedialität, also durch die Kombination von Text und Bild (Wolf 2015; Mayer 2009). Populär ist beispielsweise die Verwendung der Legetechnik. Hier werden ausgeschnittene Figuren, Abbildungen oder Texte per Hand gelegt und verschoben und meist von einem Erzähler aus dem Off kommentiert (Schön 2013). Ebenfalls weit verbreitet sind fiktive Akteure, die sich in einer Situation befinden, die jener des Betrachters ähnelt. So werden Emotionen mit Fakten verbunden und es wird ein konkreter Kontext (Situierung) geschaffen, der die Videos lebendig und informativ macht (Slopinski 2016). Eine weitere Möglichkeit stellen Bildschirmaufzeichnungen

1 Zudem sind Video-Tutorials von Formaten wie Videos beim Flipped Classroom, der Präsentation von Informationen im Zuge von Anchored Instruction oder dem Digital Storytelling abzugrenzen. 
(Screencasts) dar. Ein Screencast-Video dokumentiert und kommentiert Bildschirmgeschehen (z.B. im Zuge der Einführung einer Software). Zudem können vortragsbzw. erklärungsunterstützende Notizen oder Folien gezeigt werden (Hackfort 2016; Schaarschmidt, Albrecht und Börner 2016; Schön 2013; Wolf 2015). Die letztlich vielleicht einfachste Variante besteht darin, sich selbst mittels Webcam beim Erklären zu filmen (Schön 2013).

Videoerklärungen weisen einige Unterschiede zu herkömmlichen Instruktionsformen auf. In erster Linie ist hier die fehlende Möglichkeit, direkt (Rück-)Fragen zu stellen, zu nennen. Daher ist es von Bedeutung, Erklärvideos möglichst durch interaktive Elemente zu ergänzen, um den Lernenden die Möglichkeit zur aktiven Verarbeitung der im Video präsentierten Informationen zu eröffnen. Ohne Interaktivität haben Videos z.B. gegenüber schriftlichen Erklärungen den Nachteil, dass Betrachtende wenig Einfluss auf ihren Informationsverarbeitungsprozess haben (z.B. Inhalte zu wiederholen oder zu überspringen). Die Informationsverarbeitung wird durch die sich schnell verändernden, flüchtig präsentierten Inhalte erschwert, da Rezipierende entscheidende Inhalte ggf. nicht aufnehmen können oder Elaborationsprozesse nicht abschliessen, um nachfolgende Informationen nicht zu verpassen (Merkt et al. 2011; Merkt 2012; Merkt und Schwan 2016; Moreno und Mayer 2007). Unterschiedliche Lerngeschwindigkeiten und kognitiven Voraussetzungen der Lernenden können nicht berücksichtigt werden (Merkt et al. 2011). Verschiedene Gestaltungselemente ermöglichen eine Anpassung von Form und Inhalt des Videos an individuelle kognitive Fähigkeiten und Bedürfnisse. Diesbezüglich sind zu nennen (Bülles, FreislebenTeutscher und Buchner 2018; Cattaneo und Sauli 2017; Delen, Liew und Willson 2014; de Koning et al. 2007; Mayer und Chandler 2001; Merkt 2012; Merkt et al. 2011; Moreno und Mayer 2007; Schwan und Riempp 2004; Shelton, Warren und Archambault 2016; Zahn, Barquero und Schwan 2004):

- Kontrolle der Videowiedergabe (Lernende bestimmen Reihenfolge und Wiedergabegeschwindigkeit durch Pausieren, Überspringen, Zurückspulen etc.);

- Segmentierung (Aufteilung in kürzere Videoabschnitte);

- Inhaltsverzeichnis \& Index (eigenständige Auswahl relevanter Abschnitte);

- Zeitstrahl (Zugriff auf einzelne Abschnitt über interaktiven Zeitstrahl);

- Manipulation (Zoom, Objekte bewegen etc.);

- Hervorhebungen (Hinweis auf relevante Aspekte durch farbliche Markierung, Einblendung von Schlüsselbegriffen etc.);

- Notizen (Zuordnen von Notizen zu bestimmten Videoszenen);

- Fragen (Möglichkeit, z.B. im Experten-Chat Fragen zu stellen);

- Gruppenfunktion (Austausch mit anderen Betrachtenden über Kommentare)

- Zusatzmaterial (Verweise auf weitere Quellen z.B. durch Hyperlinks) sowie

- Evaluation (z.B. Quizfragen). 


\section{Übersicht über die gesichteten Studien zur Wirkung verschiedener Gestaltungsele- mente von Erklärvideos}

Ziel des Beitrags ist es, auf Basis empirischer Befunde Gestaltungsempfehlungen für Erklärvideos zu erarbeiten. Die Auswahl der insgesamt 24 empirischen Studien, die in die Analyse einfliessen, wurde auf Basis folgender Überlegungen getroffen: (1) Aus einer inhaltlichen Perspektive wurde auf Einschlägigkeit geachtet, d.h. der Fokus der Studien musste auf der Analyse der Wirkung einzelner Gestaltungsemelemente von Erklärvideos liegen. (2) Design der Studien: Aus methodischer Sicht wurden in erster Linie Untersuchungen mit experimentellen Designs (im Schul- oder Hochschulkontext) herangezogen (22 Studien). Ergänzend finden zudem insgesamt zwei Studien, die die Wirkung von Gestaltungselementen von Videos in Lehrangeboten (insbesondere Massive Open Online Courses, MOOCs) thematisieren, Berücksichtigung. Eine Beschränkung mit Blick auf das Alter der Studien wurde nicht vorgenommen, da es sich bei dem Forschungsfeld um eine vergleichsweise junge Thematik handelt. Gleichwohl ist zu konstatieren, dass ältere Studien vor dem Hintergrund der schnellen technischen Entwicklung ggf. bezüglich ihrer Aussagekraft mit einer gewissen Zurückhaltung interpretiert werden sollten. Tabelle 1 gibt einen Überblick über die verarbeiteten Studien sowie die gefundenen Effekte. Die Studien werden charakterisiert hinsichtlich (1) des untersuchten Gestaltungsmerkmals sowie (2) der berücksichtigten Zielgrösse (z.B. Lernerfolg, Selbstwirksamkeit etc.). In den folgenden Abschnitten steht die Wirkung folgender Gestaltungselemente im Blickpunkt:

1. interaktive Elemente,

2. Merkmale der erklärenden Person und Videoperspektive sowie

3. sonstige Designelemente (z.B. Videotypen, Videodauer).

\begin{tabular}{|l|l|c|l|}
\hline Gestaltungselement & Zielgrösse & Effekte & Studie \\
\hline $\begin{array}{l}\text { Interaktivität } \\
\text { (Notizen, Zusatzmateri- } \\
\text { al, Übungsaufgaben) }\end{array}$ & $\begin{array}{l}\text { Lernerfolg (Wiedergabe von Fakten } \\
\text { zu erneuerbaren Energien) }\end{array}$ & $\checkmark$ & $\begin{array}{l}\text { Delen, Liew und } \\
\text { Willson (2014) }\end{array}$ \\
\hline $\begin{array}{l}\text { Interaktivität } \\
\text { (Wiedergabekontrolle, } \\
\text { Segmentierung) }\end{array}$ & $\begin{array}{l}\text { Lernerfolg (Wiedergabe/Transfer; } \\
\text { offene Testfragen zur Entstehung von } \\
\text { Tag und Nacht) }\end{array}$ & $\checkmark$ & $\begin{array}{l}\text { Hasler, Kersten und } \\
\text { Sweller (2007) }\end{array}$ \\
\hline $\begin{array}{l}\text { Interaktivität } \\
\text { (Segmentierung) }\end{array}$ & $\begin{array}{l}\text { Lernerfolg (Wiedergabe/Transfer; } \\
\text { offene Testfragen zur Entstehung von } \\
\text { Blitzen) }\end{array}$ & $\checkmark$ & $\begin{array}{l}\text { Mayer und Chandler } \\
\text { (2001) }\end{array}$ \\
\hline $\begin{array}{l}\text { Interaktivität } \\
\text { (Inhaltsverzeichnis, } \\
\text { Register) }\end{array}$ & $\begin{array}{l}\text { Lernerfolg (Essay-Aufgabe zum Pots- } \\
\text { damer Abkommen) }\end{array}$ & --- & $\begin{array}{l}\text { Merkt und Schwan } \\
\text { (2014) }\end{array}$ \\
\hline $\begin{array}{l}\text { Interaktivität } \\
\text { (Wiedergabekontrolle) }\end{array}$ & Lernerfolg (Segelknoten) & $\checkmark$ & $\begin{array}{l}\text { Schwan und Riempp } \\
\text { (2004) }\end{array}$ \\
\hline $\begin{array}{l}\text { Interaktivität } \\
\text { (Segmentierung) }\end{array}$ & $\begin{array}{l}\text { Lernerfolg (Anwendungsaufgaben } \\
\text { zur Wahrscheinlichkeitsrechnung) }\end{array}$ & $\checkmark$ & Spanjers et al. (2012) \\
\hline
\end{tabular}




\begin{tabular}{|c|c|c|c|}
\hline Gestaltungselement & Zielgrösse & Effekte & Studie \\
\hline $\begin{array}{l}\text { Interaktivität } \\
\text { (Hyperlinks) }\end{array}$ & $\begin{array}{l}\text { Lernerfolg (Wiedergabe/Transfer; } \\
\text { Single Choice Items zu Seen als Öko- } \\
\text { system) }\end{array}$ & $(\sqrt{ })$ & $\begin{array}{l}\text { Zahn, Barquero und } \\
\text { Schwan (2004) }\end{array}$ \\
\hline $\begin{array}{l}\text { Interaktivität } \\
\text { (Wiedergabekontrolle, } \\
\text { Segmentierung und } \\
\text { Inhaltsverzeichnis) }\end{array}$ & $\begin{array}{l}\text { Lernerfolg (geschlossene Fragen zu } \\
\text { Online-Suchmaschinen) } \\
\text { Zufriedenheit }\end{array}$ & $\begin{array}{l}\checkmark \\
\checkmark\end{array}$ & Zhang et al. (2006) \\
\hline $\begin{array}{l}\text { Erklärende Person } \\
\text { (Sichtbarkeit) }\end{array}$ & $\begin{array}{l}\text { Lernerfolg (Anwendung/Transfer; } \\
\text { Wahrscheinlichkeitsrechnung) } \\
\text { Selbstwirksamkeit } \\
\text { wahrgenommene Kompetenz } \\
\text { Lernanstrengung } \\
\text { Freude am Lernen } \\
\text { Selbstregulation }\end{array}$ & $\begin{array}{l}-- \\
--- \\
--- \\
--- \\
--- \\
---\end{array}$ & $\begin{array}{l}\text { Hoogerheide, Loyens } \\
\text { und van Gog (2014a) }\end{array}$ \\
\hline $\begin{array}{l}\text { Erklärende Person } \\
\text { (Sichtbarkeit) }\end{array}$ & $\begin{array}{l}\text { Lernerfolg (Abschlussnote des } \\
\text { MOOC) } \\
\text { Prüfungsteilnahme } \\
\text { Schwundquote des MOOC } \\
\text { Cognitive Load } \\
\text { wahrgenommene soziale Präsenz der } \\
\text { Lehrperson }\end{array}$ & $\begin{array}{l}-- \\
--- \\
--- \\
\checkmark \\
\checkmark\end{array}$ & $\begin{array}{l}\text { Kizilcec, Bailenson } \\
\text { und Gomez (2015) }\end{array}$ \\
\hline $\begin{array}{l}\text { Erklärende Person } \\
\text { (Sichtbarkeit) }\end{array}$ & $\begin{array}{l}\text { Lernerfolg (Problemlöseaufgabe) } \\
\text { Lernanstrengung } \\
\text { Aufmerksamkeitssteuerung }\end{array}$ & $\begin{array}{l}-- \\
-- \\
\checkmark\end{array}$ & $\begin{array}{l}\text { Ouwehand, van Gog } \\
\text { und Paas (2015) }\end{array}$ \\
\hline $\begin{array}{l}\text { Erklärende Person } \\
\text { (Sichtbarkeit) }\end{array}$ & $\begin{array}{l}\text { Lernerfolg (Problemlöseaufgabe; } \\
\text { Anwendung und } \\
\text { Transfer) }\end{array}$ & $\begin{array}{c}\checkmark \\
---\end{array}$ & $\begin{array}{l}\text { van Gog, I. Verveer } \\
\text { und L. Verveer (2014) }\end{array}$ \\
\hline $\begin{array}{l}\text { Erklärende Person } \\
\text { (Sichtbarkeit) }\end{array}$ & $\begin{array}{l}\text { Lernerfolg (Wiedergabe von Fakten- } \\
\text { wissen über Moleküle und Zusam- } \\
\text { mensetzen von Molekülen) }\end{array}$ & --- & $\begin{array}{l}\text { van Wermeskerken, } \\
\text { Grimmius und van } \\
\text { Gog (2018) }\end{array}$ \\
\hline $\begin{array}{l}\text { Erklärende Person } \\
\text { (Sichtbarkeit) }\end{array}$ & $\begin{array}{l}\text { Lernerfolg (Anwendung/Transfer; } \\
\text { Wahrscheinlichkeitsrechnung) }\end{array}$ & --- & $\begin{array}{l}\text { van Wermeskerken, } \\
\text { Ravensbergen und } \\
\text { van Gog (2017) }\end{array}$ \\
\hline $\begin{array}{l}\text { Erklärende Person } \\
\text { (Sichtbarkeit) }\end{array}$ & $\begin{array}{l}\text { Lernerfolg (Wiedergabe von Fakten- } \\
\text { wissen über Atome und Zusammen- } \\
\text { setzen eines Atommodells) }\end{array}$ & --- & $\begin{array}{l}\text { van Wermeskerken } \\
\text { und van Gog (2017) }\end{array}$ \\
\hline $\begin{array}{l}\text { Erklärende Person } \\
\text { (Expertise, Alter) }\end{array}$ & $\begin{array}{l}\text { Lernerfolg (Anwendungsaufgaben zu } \\
\text { elektrischen Schaltungen) } \\
\text { wahrgenommene Kompetenz } \\
\text { Selbstwirksamkeit } \\
\text { Lernanstrengung } \\
\text { Freude am Lernen } \\
\text { wahrgenommene Erklärungsqualität }\end{array}$ & $\begin{array}{l}(\checkmark) \\
--- \\
-- \\
(\checkmark) \\
-- \\
\checkmark\end{array}$ & $\begin{array}{l}\text { Hoogerheide et al. } \\
(2016 b)\end{array}$ \\
\hline $\begin{array}{l}\text { Erklärende Person } \\
\text { (Geschlecht) }\end{array}$ & $\begin{array}{l}\text { Lernerfolg (Anwendung/Transfer; } \\
\text { Wahrscheinlichkeitsrechnung) } \\
\text { wahrgenommene Kompetenz } \\
\text { Selbstwirksamkeit } \\
\text { Lernanstrengung } \\
\text { Freunde am Lernen }\end{array}$ & $\begin{array}{l}\checkmark \\
--- \\
\checkmark \\
\checkmark\end{array}$ & $\begin{array}{l}\text { Hoogerheide, Loyens } \\
\text { und van Gog (2016) }\end{array}$ \\
\hline
\end{tabular}




\begin{tabular}{|c|c|c|c|}
\hline Gestaltungselement & Zielgrösse & Effekte & Studie \\
\hline $\begin{array}{l}\text { Erklärende Person } \\
\text { (Geschlecht) }\end{array}$ & $\begin{array}{l}\text { Lernerfolg (Anwendungsaufgaben zu } \\
\text { elektrischen Schaltungen) } \\
\text { wahrgenommene Kompetenz } \\
\text { Selbstwirksamkeit } \\
\text { Lernanstrengung } \\
\text { Freude am Lernen } \\
\text { wahrgenommene Erklärungsqualität }\end{array}$ & $\begin{array}{l}--- \\
--- \\
--- \\
--- \\
--- \\
---\end{array}$ & $\begin{array}{l}\text { Hoogerheide et al. } \\
(2018)\end{array}$ \\
\hline Videoperspektive & $\begin{array}{l}\text { Lernerfolg (Aufbau elektrischer } \\
\text { Schaltkreise) }\end{array}$ & $\checkmark$ & Fiorella et al. (2017) \\
\hline Videotypen & $\begin{array}{l}\text { Lernerfolg (Wiedergabe/Verständnis/ } \\
\text { Anwendung) } \\
\text { Aufmerksamkeit } \\
\text { Emotionen } \\
\text { Cognitive Load }\end{array}$ & $\begin{array}{l}\checkmark \\
\checkmark \\
-- \\
\checkmark\end{array}$ & Chen und Wu (2015) \\
\hline $\begin{array}{l}\text { Dauer } \\
\text { Sichtbarkeit (Erklären- } \\
\text { de/r) } \\
\text { Sprechgeschwindig- } \\
\text { keit/Enthusiasmus } \\
\text { Videotyp } \\
\text { Produktionsstil }\end{array}$ & $\begin{array}{l}\text { Engagement (Dauer der Beschäfti- } \\
\text { gung mit dem Video und Bearbeitung } \\
\text { von Verständnisaufgaben) }\end{array}$ & $\begin{array}{l}\checkmark \\
\checkmark \\
\checkmark \\
\checkmark \\
\checkmark\end{array}$ & $\begin{array}{l}\text { Guo, Kim und Rubin } \\
\text { (2014) }\end{array}$ \\
\hline $\begin{array}{l}\text { Objektive Ästhetik und } \\
\text { Nutzerfreundlichkeit, } \\
\text { Wahrgenommene } \\
\text { Ästhetik und Nutzer- } \\
\text { freundlichkeit }\end{array}$ & Emotion ( $\rightarrow$ Lernerfolg, Motivation) & $\checkmark$ & $\begin{array}{l}\text { Heidig, Müller und } \\
\text { Reichelt (2015) }\end{array}$ \\
\hline Videotypen & $\begin{array}{l}\text { Lernerfolg (Wiedergabe von Fakten } \\
\text { zur US Präsidentschaftswahl) }\end{array}$ & $\checkmark$ & $\begin{array}{l}\text { Krämer und Böhrs } \\
(2017)\end{array}$ \\
\hline Emotionales Design & $\begin{array}{l}\text { Positive Emotionen (u.a. Interesse, } \\
\text { Enthusiasmus) } \\
\text { Lernerfolg (Wiedergabe und Anwen- } \\
\text { dung; Immunisierung) } \\
\text { Lernerfolg (Selbsteinschätzung) } \\
\text { Cognitive Load } \\
\text { Zufriedenheit } \\
\text { Motivation }\end{array}$ & $\begin{array}{l}\checkmark \\
\checkmark \\
--- \\
--- \\
---\end{array}$ & Plass et al. (2014) \\
\hline
\end{tabular}

Tab. 1.: Übersicht über die gesichteten Studien.

Anmerkung: $\checkmark$ = positiver Effekt, $(\checkmark)$ = bedingt positiver Effekt, --- = kein Effekt.

\section{Bedeutung interaktiver Elemente}

Empirische Befunde schreiben insbesondere jenen Erklärvideos, die interaktive Elemente beinhalten, positive Lerneffekte zu. Von den acht gesichteten Studien, welche die Wirkung interaktiver Elemente untersuchten, identifizieren sieben positive Lerneffekte. Die Studien analysieren dabei unterschiedliche interaktive Elemente. Beispielsweise untersuchen Zhang et al. (2006) die Wirkung von Erklärvideos, welche verschiedene interaktive Elemente vereinen (Wiedergabekontrolle, Segmentierung, 
Inhaltsverzeichnis). Die Autoren vergleichen vier Szenarien einer Lernumgebung für Studierende: (1) E-Learning mit interaktiven Videos (Möglichkeit der Wiedergabekontrolle, Segmentierung und Inhaltsverzeichnis), (2) E-Learning mit nicht-interaktiven Videos, (3) E-Learning ohne Videos, (4) traditionelle Vorlesung. Die Befunde zeigen, dass Studierende, die interaktive Videos nutzten, im Vergleich zu den drei anderen Gruppen deutlich besseren Lernerfolg erzielen. Unterschiede zwischen der Gruppe mit nicht-interaktiven Videos und jener ohne Videos sind dagegen nicht auszumachen. Darüber hinaus zeigen sich auf Basis von Selbstberichtsdaten signifikante Unterschiede hinsichtlich der Zufriedenheit; diese fällt in der Gruppe der Studierenden, die interaktive Videos nutzen, signifikant höher aus als in allen anderen Gruppen. In einem ähnlichen Setting identifizieren Delen, Liew und Willson (2014) ebenfalls signifikant bessere Lernergebnisse für Studierende, die interaktive Videos nutzen (interaktive Elemente: Notizen, Zusatzmaterial, Übungsaufgaben; Kontrollgruppe: Videos ohne interaktive Elemente). Dies gilt auch, wenn für die Zeit kontrolliert wird, welche die Studierenden für die Auseinandersetzung mit dem Videomaterial aufwenden.

Darüber hinaus liegen vier Studien vor, die sich speziell mit der Segmentierung von Videos befassen (eine Form interaktiver Elemente, s.o.). Diesbezüglich besteht prinzipiell die Möglichkeit, im Video entweder feste Pausen zu implementieren, die sich an der Strukturierung der Lerninhalte orientieren oder aber den Lernenden das Setzen von Pausen an beliebigen Stellen (Start, Stopp, Spulen) zu ermöglichen. Vorliegende Studien zeigen, dass beide Methoden zu lernwirksamen Effekten führen. Beispielsweise erreichen in der Studie von Mayer und Chandler (2001) Studierende, die ein vorsegmentiertes Video betrachten (16 einzelne Inhaltsabschnitte), bessere Lernergebnisse als die Kontrollgruppe, welcher das Video ohne Unterbrechungen präsentiert wurde. Darüber hinaus zeigen die Befunde von Schwan und Riempp (2004), dass Studierende, welche die Möglichkeit der Wiedergabesteuerung nutzen konnten, weniger Zeit benötigten, um auf Basis von Videos eine Fähigkeit zu erlernen (hier: Binden von Seemannsknoten). Hasler, Kersten und Sweller (2007) verknüpfen die Segmentierung der Videoinhalte mit der Möglichkeit der Wiedergabesteuerung durch Lernende. Dabei konnten die Schülerinnen und Schüler entweder elf verschiedene vorgegebene Videosequenzen jeweils selbst starten oder aber das Video selbstständig abspielen und an beliebigen Stellen pausieren. Beide Experimentalgruppen erzielten bessere Lernergebnisse im Vergleich zu Schülerinnen und Schülern, denen keine Möglichkeit zur Wiedergabekontrolle eingeräumt wurde. Zwischen den beiden Experimentalgruppen konnten keine Unterschiede festgestellt werden. Zudem wurde von der Möglichkeit, selbst Pausen zu setzen, kaum Gebrauch gemacht. Hasler, Kersten und Sweller (2007) begründen die dennoch positiven Lerneffekte der freien Wiedergabesteuerung damit, dass die Lernenden - während sie sinnvolle Stellen für Pausen suchten - das Videomaterial aufmerksamer betrachten und hinsichtlich einer inhaltlichen Strukturierung analysierten, wodurch eine tiefere Verarbeitung der 
präsentierten Inhalte erreicht wurde. Folglich scheint es eher auf die Strukturierung der Inhalte anzukommen und weniger darauf, ob diese durch die Lernenden (implizit) vorgenommen werden kann oder bereits (explizit) vorliegt.

Warum genau sich die Lernergebnisse bei einer Segmentierung der Inhalte verbessern, ist indes noch nicht abschliessend geklärt. Es liegen zwei mögliche Erklärungsansätze vor, die sich nicht unbedingt gegenseitig ausschliessen müssen (vgl. Spanjers et al. 2012). Einerseits könnte durch Pausen zwischen einzelnen Erklärungsinhalten eine Strukturierung ersichtlich werden, d.h. die positiven Lerneffekte resultieren aus der Verdeutlichung der Struktur des Fachinhalts. Andererseits wird davon ausgegangen, dass insbesondere die Pausen zwischen Lerneinheiten entscheidend sind, da Lernenden somit zusätzliche Zeit für kognitive Prozesse zur Verfügung steht. Die Lernenden müssen ihre Aufmerksamkeit nicht zwischen dem Verarbeiten gerade rezipierter Informationen und der Aufnahme neuer Informationen teilen. Es ist ebenfalls denkbar, dass die Effekte aus der Kombination beider Effekte resultieren (Merkt und Schwan 2016). Spanjers et al. (2012) zeigen, dass kleine Pausen zwischen einzelnen Inhaltssegmenten eines Videos die Lernleistung von Schülerinnen und Schülern positiv beeinflussen. Zusätzlich reduzieren visuelle Hinweise auf eine Segmentierung (Verdunkeln des Bildschirms zwischen einzelnen Videosegmenten) den Cognitive Load der Lernenden, allerdings ohne die Lernleistung zu beeinflussen. Folglich scheinen beide Effekte eine gewisse Rolle zu spielen. Allerdings konnten diese nicht komplett voneinander isoliert werden, da Pausen automatisch auch eine Strukturierung vorgeben.

Eine weitere Möglichkeit, Videos interaktiv zu gestalten, besteht in der Implementierung einer Suchfunktion über Inhaltsverzeichnisse und Register. Zur Analyse der Effekte solcher Steuerungselemente untersuchen Merkt und Schwan (2014) vier Gruppen von Studierenden, die in unterschiedlichen Lernumgebungen vor die Aufgabe gestellt werden, einen Essay über die Lerninhalte zu verfassen. Die Befunde zeigen, dass Studierende, welche die Suchfunktionen nutzen konnten, eine grössere Anzahl relevanter Fakten identifizierten als jene, denen Videos ohne Inhaltsverzeichnisse und Register zur Verfügung standen. Die Nutzung eines entsprechenden Lehrbuchs führt jedoch zu einer vergleichbaren Anzahl replizierter Fakten, so dass sich die Vorteile des Videos wieder relativieren. Darüber hinaus zeigt sich, dass die interaktiven Elemente lediglich für jene Inhaltsaspekte Vorteile bieten, welche direkt im Register angesprochen oder durch dieses angewählt werden konnten. Die Gesamtqualität der Essays war in der Gruppe der Lernenden mit interaktiven Videos nicht höher als in den anderen Untersuchungsgruppen.

Schliesslich untersuchen Zahn, Barquero und Schwan (2004) die Wirkung von Hyperlinks, über welche Zusatzinformationen zum Videomaterial bereitgestellt werden. Hinsichtlich der Lerneffekte von Untersuchungsgruppen, die Videos mit Hyperlinks nutzten und jenen, die keine Hyperlinks zur Verfügung hatten, zeigen sich keine 
signifikanten Unterschiede. Der Wissenszuwachs zwischen Pre- und Posttest fällt für die verschiedenen Gruppen ähnlich hoch aus. Betrachtet man lediglich die Teilnehmenden, denen Hyperlinks zur Verfügung gestellt wurden, zeigt sich allerdings, dass sich eine intensivere Nutzung der Links und eine längere Beschäftigung mit dem Zusatzmaterial positiv auf die Lernleistung auswirkten.

\section{Bedeutung der erklärenden Person und der Videoperspektive}

Im Folgenden wird der Frage nachgegangen, ob Videos mit menschlichen Modellen (Präsenz der erklärenden Person) solchen mit reiner Text- und Bilddarstellung grundsätzlich überlegen sind. Zunächst ist davon auszugehen, dass das Gesicht des Erklärenden Aufmerksamkeit auf sich zieht. Eye-Tracking-Studien zu sozialen Interaktionen zeigten, dass Menschen etwa $90 \%$ der Zeit einer Person, die etwas erklärt, ins Gesicht schauen, und zwar unabhängig davon, ob es sich um eine reale Person oder einer Person in einem Video handelt, welche die Blickrichtung des Betrachters oder der Betrachterin gar nicht wahrnehmen kann (Gullberg und Holmqvist 2006). Folglich bietet das Gesicht des Erklärenden einerseits ein gewisses Ablenkungspotenzial vom Lerninhalt. Andererseits könnte insbesondere in Tutorials, in denen eine Handlung demonstriert und erklärt wird, zusätzlich zu den Bewegungen der Hände auch die Blickrichtung des Erklärenden unterstützend wirken, da Menschen typischerweise den Blicken eines anderen Menschen, mit dem sie interagieren, folgen (u. a. Driver et al. 1999).

Die gesichteten empirischen Studien können mehrheitlich keine signifikanten Effekte der Sichtbarkeit des/r Erklärenden nachweisen. Signifikante Effekte für den Lernerfolg werden lediglich in einer von sieben Studien identifiziert. In einem experimentellen Design legen van Gog, I. Verveer und L. Verveer (2014) Lernenden eines von zwei Videos mit Problemlöseaufgaben vor, wobei bei beiden Videos die Hände und ein Teil des Oberkörpers des Erklärenden sichtbar sind, jedoch nur bei einem Video auch das Gesicht (Experimentalgruppe). Die Teilnehmenden der Experimentalgruppe schneiden bei einer sich an das Video anschliessenden Bearbeitung der gleichen Aufgabe (nicht jedoch bei einer Transferaufgabe) besser ab als jene der Kontrollgruppe. Beide Gruppen blicken während der Betrachtung des Videos hauptsächlich auf den Bereich, in dem die Demonstration stattfindet. Das gelegentliche Betrachten des Gesichts scheint der Experimentalgruppe allerdings zusätzliche Hinweise auf besonders relevante Aspekte zu geben. Allerdings konnten diese Ergebnisse in nachfolgenden Studien, die Videos mit bzw. ohne sichtbares Gesicht der erklärenden Person vergleichen, nicht repliziert werden (Hoogerheide, Loyens und van Gog 2014a; Ouwehand, van Gog und Paas 2015; van Wermeskerken, Grimmius und van Gog 2018; van Wermeskerken, Ravensbergen und van Gog 2017; van Wermeskerken und van Gog 2017). Ouwehand, van Gog und Paas (2015) zeigen zwar, dass Blicke und 
Gesten der erklärenden Person die Aufmerksamkeit gezielt auf relevante Aspekte des Erklärgegenstandes lenken können, allerdings wirkte sich die Lenkung der Aufmerksamkeit auch in der Studie ebenfalls nicht signifikant auf die Lernanstrengung oder den Lernerfolg aus. Im Vergleich zweier Formate eines MOOCs (untersuchte Formate: konstante Sichtbarkeit der Lehrperson vs. strategisches Einsetzen des Bildes der Lehrperson an inhaltlich passenden Stellen) konnten auch Kizilcec, Bailenson und Gomez (2015) keine Effekte für den Lernerfolg im Kurs feststellen. Schliesslich scheinen nicht-kognitive Merkmale durch die Sichtbarkeit der erklärenden Person ebenfalls nicht beeinflusst zu werden. Hoogerheide, Loyens und van Gog (2014a) untersuchen drei unterschiedliche Lernsituationen: (1) Video mit sichtbarem Gesicht des Modells, Aufgabe wird demonstriert; (2) Video ohne sichtbares Gesicht, mündliche Erklärung, Aufgabe in Bildern; (3) Text und Bilder ohne Modell (Lösungsbeispiel). Es zeigen sich weder Unterschiede in Bezug auf den Lernerfolg noch in Bezug auf Selbstwirksamkeit, Lernanstrengung, Lernfreude oder Selbstregulation. Lediglich Guo, Kim und Rubin (2014) zeigen, dass sich die Sichtbarkeit des Lehrenden auf das Engagement der Lernenden in MOOCs (Dauer der Beschäftigung mit dem Video und Bearbeitung von Verständnisaufgaben) auswirkt (nähere Beschreibung der Studie erfolgt im folgenden Abschnitt).

Für den Fall, dass der/die Erklärende im Video dargestellt ist, ist zusätzlich von Interesse, inwiefern die Perspektive der Videoaufnahme die Lernleistung beeinflusst. Bei der Wahl der Perspektive gibt es für Video-Tutorials prinzipiell zwei Optionen: Das Video kann (1) aus der Sicht des Erklärenden (Modell) oder (2) einer Person, die das Modell beobachtet, gefilmt werden. Prinzipiell wird davon ausgegangen, dass beim Nachmachen einer Handlung ein geringerer Cognitive Load auftritt, wenn das Video aus der Sicht der vormachenden Person gefilmt wurde und die Lernenden keinen Perspektivenwechsel vornehmen müssen, da die Handgriffe bereits aus jener Perspektive dargeboten werden, die sie selbst bei der Ausführung einnehmen. Die Befunde von Fiorella et al. (2017) bestätigen dies. Hier erzielen Studierende, die den Aufbau eines Schaltkreises aus der Sicht des Erklärenden erlernen bessere Lernergebnisse. Dabei zeigen sich mit steigender Komplexität der erlernten Aufgabe auch umso stärkere Effekte der Perspektive.

Schliesslich liegen drei Studien vor, die untersuchen, welche Relevanz bestimmten Eigenschaften des Modells zukommt. Gemäss der Model-Observer-Similarity-Hypothese (Schunk 1987) moderieren Ähnlichkeiten zwischen Lernenden und Modell (z.B. Alter, Geschlecht) die Lernerfolge der Zuhörenden. Hoogerheide et al. (2016b) untersuchen den Einfluss der Expertise bzw. des Alters des Erklärenden. In einem Studiendesign mit vier Gruppen betrachten Schülerinnen und Schüler jeweils ein Video, in dem entweder ein gleichaltriges Modell oder eine Erwachsene den Aufbau elektrischer Schaltungen erklärt. Dem Modell wird im Vorfeld zusätzlich entweder eine hohe oder geringe Expertise zugeschrieben. Die Videos präsentierten abgesehen 
von der Einführung jedoch identische Erklärungen. Die Befunde zeigten keinerlei Effekte der vermeintlichen Expertise auf den Lernerfolg, die Lernanstrengung, die Selbstwirksamkeit, die wahrgenommene Kompetenz oder die Lernfreude. Allerdings wurde den Erklärungen der vermeintlichen Experten eine höhere Qualität zugeschrieben. Zudem wirkte sich das Alter des Modells positiv auf den Lernerfolg, die Lernanstrengung und die wahrgenommene Erklärungsqualität aus.

In Bezug auf das Geschlecht der erklärenden Person sind die empirischen Befunde erneut nicht eindeutig. Hoogerheide et al. (2018) können weder für kognitive Merkmale (Lernerfolg) noch für nicht-kognitive Merkmale (Selbstwirksamkeit, Lernanstrengung, Lernfreude, wahrgenommene Erklärungsqualität) signifikante Effekte des Geschlechts des Erklärenden nachweisen. Dies gilt, obwohl die Teilnehmenden den Erklärungsinhalt (Aufbau elektrischer Schaltkreise) als eher passend für männliche Modelle erachteten. In der Studie von Hoogerheide, Loyens und van Gog (2016) wirkt sich das Geschlecht des Erklärenden ebenfalls nicht auf die Lernergebnisse aus. Allerdings zeigen sich vereinzelt Effekte hinsichtlich der wahrgenommenen eigenen Kompetenz (stärkerer Zuwachs von Pre- zu Post-Test bei der Betrachtung eines männlichen Modells). Schliesslich berichten männliche Lernende bei der Betrachtung männlicher Erklärender höhere Lernfreude und geringere Lernanstrengungen als weibliche Lernende, die das gleiche Video nutzten.

\section{Bedeutung sonstiger Designelemente}

Weitere fünf empirische Studien befassen sich mit sonstigen Designelementen von Erklärvideos. Zunächst können verschiedene Videotypen in den Blick genommen werden. Hier finden Chen und Wu (2015) im Vergleich von drei verschiedenen Typen (Vorlesungsaufzeichnung, Screencast, Bild-in-Bild-Variante) signifikante Unterschiede hinsichtlich Aufmerksamkeit, Cognitive Load und Lernerfolg. Während Screencast-Videos eine höhere Aufmerksamkeit der Lernenden erreichen - gleichzeitig allerdings einen höheren Cognitive Load erfordern - schneiden die Vorlesungsaufzeichnung sowie die Bild-in-Bild-Variante hinsichtlich des Lernerfolgs besser ab. Bezüglich der ausgelösten Emotionen lassen sich hingegen keine bedeutsamen Unterschiede zwischen den verschiedenen Videotypen feststellen. Auch in der Studie von Krämer und Böhrs (2017) ergeben sich für verschiedene Erklärvideos zum selben Thema Unterschiede in der Lernleistung der Teilnehmenden.

In einer umfangreichen Studie (6,9 Millionen Video-Sessions im Rahmen von MOOCs) untersuchen Guo, Kim und Rubin (2014) das Engagement der Lernenden, welches sie einerseits über die Zeit, in der sich Lernende mit einem Erklärvideo beschäftigen, erfassten sowie andererseits über die Bearbeitung eines Problems im Anschluss an das Betrachten des Videos (der Versuch, diese Anwendungsaufgabe zu lösen, wird als höheres Engagement gewertet als das Überspringen der Aufgabe). Auch 
hier lassen sich Unterschiede zwischen verschiedenen Videotypen identifizieren. Das Engagement der Lernenden erweist sich bei Videos, in denen Aufzeichnungen auf einem Tablet gemacht werden, als signifikant höher als bei Videos, die Vorlesungsfolien oder Screencasts zeigen. Darüber hinaus lassen sich keine Hinweise auf eine Überlegenheit professioneller Studioproduktionen finden. Vielmehr scheint sich ein informelles Setting positiv auszuwirken. Schliesslich wirkt eine hohe Sprechgeschwindigkeit - welche mit einer enthusiastischeren Darbietung einhergeht - positiv auf das Engagement. Als bedeutsamsten Indikator für das Engagement der Lernenden identifizieren Guo, Kim und Rubin (2014) die Länge des Videos. Längere Videos werden häufiger abgebrochen, sich anschliessende Aufgaben seltener bearbeitet. Die Autoren empfehlen daher eine maximale Dauer von sechs Minuten für einzelne Erklärvideos. Längere Einheiten sollten sinnvoll auf mehrere Videos aufgeteilt werden. Die Befunde zur Videolänge decken sich wiederum mit der Befragung von Krämer und Böhrs (2017). Hier kommt der Länge verschiedener Videos eine signifikante Rolle für deren Beurteilung durch die Lernenden zu.

Schliesslich untersuchen Plass et al. (2014) Effekte eines «emotionalen Designs» einer multimedialen Lernumgebung. Die Annahme war, dass ein entsprechendes Design positive Emotionen bei Lernenden auslösen kann, die wiederum den Cognitive Load und die Lernergebnisse beeinflussen sowie Motivation, Zufriedenheit und Wahrnehmung der Aufgabenschwierigkeit fördern. Die Ergebnisse zeigen, dass bestimmte Designelemente sich auf Emotionen und Lernleistung der Teilnehmenden auswirken. Motivation, Cognitive Load sowie Zufriedenheit und wahrgenommener Lernerfolg bleiben jedoch unbeeinflusst. In einer Folgestudie finden Heidig, Müller und Reichelt (2015) hingegen keinen Einfluss objektiver Unterschiede in der Ästhetik der Lernmaterialien oder der Benutzerfreundlichkeit der multimedialen Lernumgebung auf das emotionale Befinden der Lernenden. Jedoch beeinflusste die subjektiv wahrgenommene Ästhetik und die Nutzerfreundlichkeit das emotionale Befinden. Dieses hat wiederum Einfluss auf die intrinsische Motivation der Lernenden, darunter auch die Bereitschaft, längerfristig mit dem Material zu arbeiten.

\section{Diskussion}

Empirische Befunde dokumentieren positive Effekte einer Rezeption von Erklärvideos - sowohl für kognitive als auch für nicht-kognitive Merkmale (z.B. Lernmotivation, Zufriedenheit). Das Ziel des vorliegenden Beitrags war eine Analyse der Wirkung verschiedener Designmerkmale und die Ableitung von Gestaltungsempfehlungen für Erklärvideos. Auf Basis der rezipierten Studien erweisen sich folgende Elemente als bedeutsam: 
Einsatz interaktiver Elemente

Videoperspektive

Design Person
Die Möglichkeit, interaktive Elemente zu nutzen, wirkt sich positiv auf den Lernerfolg aus. Den Lernenden wird eine aktive und individuelle Verarbeitung der Videoinhalte ermöglicht, indem die Erklärung an unterschiedliche Lerngeschwindigkeiten oder kognitive Voraussetzungen angepasst werden kann. Hierdurch wird ein zentraler Nachteil von Videoerklärungen ausgeglichen - die fehlende Abstimmung auf den individuellen Adressaten.

Bei der Demonstration einer Handlung - insbesondere einer komplexen Handlung - sollte das Video aus Sicht des Erklärenden gedreht werden. Beim Nachmachen der Handlung wird so der Cognitive Load des Lernenden reduziert, da der Perspektivenwechsel entfällt.

Alter der erklärenden Die Betrachtung eines Videos mit älteren Erklärenden wirkt sich im Vergleich zu Peer-Erklärungen positiv auf den Lernerfolg, die Lernanstrengung und die Beurteilung der Erklärungsqualität aus. Lernende scheinen älteren Erklärenden eine höhere Expertise zuzuschreiben und ihren Erklärungen mehr Aufmerksamkeit zu widmen.

Durch die Vielzahl an Informationen, die in einem Video auf unterschiedlichen Kanälen transportiert werden, ist es sinnvoll, die Länge der Darbietung einzuschränken. Empirische Befunde legen eine maximale Länge von sechs Minuten nahe.

Es zeigen sich signifikante lernwirksame Effekte eines Lernumgebungsdesigns, welches positive Emotionen auslöst. Zudem wirkt sich die wahrgenommene Ästhetik und Nutzerfreundlichkeit des Designs auf die Emotionen, die Motivation und den Lernerfolg der Lernenden aus.

In Bezug auf verschiedene Videotypen ergeben sich ebenfalls signifikante Effekte. Im Vergleich zu Vorlesungsaufzeichnung sowie die Bild-in-Bild-Videos führen Screencasts zu höherer Aufmerksamkeit der Lernenden - gleichzeitig jedoch zu höherem Cognitive Load und geringerem Lernerfolg. Das Engagement der Lernenden ist bei Screencasts hingegen signifikant geringer als bei Videos, in denen Aufzeichnungen auf einem Tablet gemacht werden. Letztendlich kann auf Basis der vorliegenden Befunde keine abschliessende Empfehlung für die Verwendung von Videotypen ausgesprochen werden. Diese sollte sich an den Erklärinhalten bzw. den Lernzielen orientieren. Schliesslich lassen sich bezüglich der Sichtbarkeit der erklärenden Person mehrheitlich keine signifikanten Effekte für den Lernerfolg feststellen. Gleiches gilt für das Geschlecht der Erklärenden - hier zeigen sich lediglich vereinzelte Effekte im nicht-kognitiven Bereich.

Insgesamt bieten die berichteten Befunde einschlägiger Studien also gewisse Orientierung bezüglich der Gestaltung von Erklärvideos. Allerdings beziehen sich diese lediglich auf eine Facette des Videoeinsatzes, nämlich die Rezeption von Erklärvideos durch Lernende. Insbesondere für unterrichtliche Zwecke ist darüber hinaus die (gemeinsame) Produktion von Erklärvideos von hoher Bedeutung. Wie empirische Studien zeigen, wirkt sich das Erstellen von Erklärvideos positiv auf den Wissenserwerb aus (z.B. Fiorella und Mayer 2013, 2014; Hoogerheide et al. 2016a). Werden Videos 
schliesslich gemeinsam in einer Gruppe erstellt, fördert dies zudem beispielsweise die Lernmotivation oder das Autonomieerleben der Lernenden (Slopinski 2016).

Ungeachtet des hohen Potenzials von Erklärvideos besteht jedoch hinsichtlich des Einsatzes im Unterricht noch eine Reihe von Hemmnissen. Beispielsweise behindern organisatorische Rahmenbedingungen den Einsatz von Videos. So lässt die technische Ausstattung der Schulen (ausreichende Anzahl an PCs, passende Internetbandbreite) nach wie vor zu wünschen übrig (Knaus und Valentin 2016; Lübcke und Burchert 2014; Rummler und Wolf 2012; Rummler 2017). Zudem erschwert die zeitliche Organisation (45- bzw. 90-minütige Taktung der Unterrichtsstunden) insbesondere Lernsettings, in denen die Produktion von Videos im Mittelpunkt steht. Vor diesem Hintergrund werden Medienprojekte überwiegend in Projektwochen durchgeführt, wodurch Videos einen Ausnahmestatus erhalten (Lübcke und Burchert 2014). Nicht zu unterschätzen sind darüber hinaus rechtliche Unsicherheiten, insbesondere in Bezug auf datenschutzrechtliche Bestimmungen (Knaus und Valentin 2016; Lübcke und Burchert 2014; Rummler und Wolf 2012; Rummler 2017).

Forschungsdesiderata beziehen sich insbesondere auf die Effektivität von Erklärvideos. Diese hängt zu einem grossen Teil davon ab, wie Videos in Lernumgebungen eingebettet werden und welche Verbindungen zu anderen Lernmaterialien (z.B. Aufgaben) bestehen (Karppinen 2005). Interaktivität und hypermediale Medien spielen hierbei eine bedeutende Rolle. Von Interesse wäre schliesslich, den Lernerfolg von Lernenden, die über einen längeren Zeitraum mit Videos gelernt haben, mit jenem von Lernenden zu vergleichen, die zum gleichen Thema herkömmlich unterrichtet wurden. Zusätzliche Forschung wäre auch im Bereich einer (bisher eher vernachlässigten) weiteren Form der Arbeit mit Videos wünschenswert. Denn neben Rezeption und Produktion stellt die Annotation, die Bearbeitung, Kommentierung und Veränderung von Videos, eine vielversprechende Möglichkeit der aktiven Auseinandersetzung mit Lerninhalten dar. Auch diesbezüglich stehen Forschungsbemühungen noch aus.

\section{Literatur}

Ball, Deborah Loewenberg, Heather C. Hill, und Hyman Bass. 2005. «Knowing mathematics for teaching: Who knows mathematics well enough to teach third grade, and how can we decide?» American Educator 29 (1): 14-17, 20-22, 43-46.

Bandura, Albert. 1977. Social learning theory. Eaglewood Cliffs: Prentice-Hall.

Bandura, Albert. 1986. Social foundations of thought and action: A social cognitive theory. Englewood Cliffs: Prentice-Hall.

Bitkom. 2017. Nachhilfe im Netz: Steigende Nutzerzahlen für Video-Tutorials. https://www. bitkom.org/Presse/Presseinformation/Nachhilfe-im-Netz-Steigende-Nutzerzahlen-fuerVideo-Tutorials.html. 
Bülles, Oliver, Christian F. Freisleben-Teutscher, und Josef Buchner. 2018. «Potentiale interaktiver Videos für das Inverted Classroom Model». In Inverted Classroom: Begleitband zur 7. Konferenz Inverted Classroom and Beyond 2018, herausgegeben von Josef Buchner, Christian F. Freisleben-Teutscher, Johann Haag, und Erwin Rauscher, 67-75.

Cattaneo, Alberto, und Florinda Sauli. 2017. Die Integration interaktiver Videos in didaktische Szenarien. Leitlinien des Projekts IV4VET.

Chen, Chih-Ming, und Chung-Hsin Wu. 2015. «Effects of different video lecture types on sustained attention, emotion, cognitive load, and learning performance». Computers \& Education 80: 108-121. https://doi.org/10.1016/j.compedu.2014.08.015.

Delen, Erhan, Jeffrey Liew, und Victor Willson. 2014. «Effects of interactivity and instructional scaffolding on learning: Self-regulation in online video-based environments». Computers \& Education 78: 312-320. https://doi.org/10.1016/j.compedu.2014.06.018.

Driver, Jon, Greg Davis, Paola Ricciardelli, Polly Kidd, Emma Maxwell, und Simon Baron-Cohen. 1999. "Gaze perception triggers reflexive visuospatial orienting». Visual Cognition 6 (5): 509-540. https://doi.org/10.1080/135062899394920.

Findeisen, Stefanie. 2017. Fachdidaktische Kompetenzen angehender Lehrpersonen. Wiesbaden: Springer Fachmedien Wiesbaden. https://doi.org/10.1007/978-3-658-18390-5.

Fiorella, Logan, Tamara van Gog, Vincent Hoogerheide, und Richard E. Mayer. 2017. «It's all a matter of perspective: Viewing first-person video modeling examples promotes learning of an assembly task». Journal of Educational Psychology 109 (5): 653-665. https://doi. org/10.1037/edu0000161.

Fiorella, Logan, und Richard E. Mayer. 2013. «The relative benefits of learning by teaching and teaching expectancy». Contemporary Educational Psychology 38 (4): 281-288. https://doi. org/10.1016/j.cedpsych.2013.06.001.

Fiorella, Logan, und Richard E. Mayer. 2014. «Role of expectations and explanations in learning by teaching». Contemporary Educational Psychology 39 (2): 75-85. https://doi. org/10.1016/j.cedpsych.2014.01.001.

Gog, Tamara van, Ilse Verveer, und Lise Verveer. 2014. "Learning from video modeling examples: Effects of seeing the human model's face». Computers \& Education 72: 323-327. https:// doi.org/10.1016/j.compedu.2013.12.004.

Gullberg, Marianne, und Kenneth Holmqvist. 2006. «What speakers do and what addressees look at: Visual attention to gestures in human interaction live and on video». Pragmatics \& Cognition 14 (1): 53-82. https://doi.org/10.1075/pc.14.1.05gul.

Guo, Philip J., Juho Kim, und Rob Rubin. 2014. «How video production affects student engagement: An empirical study of MOOC videos». In Proceedings of the first ACM conference on Learning @ scale conference - L@S '14, herausgegeben von Mehran Sahami, Armando Fox, Marti A. Hearst, und Michelene T. H. Chi, 41-50. New York: ACM Press. https://doi. $\operatorname{org} / 10.1145 / 2556325.2566239$.

Hackfort, Sarah. 2016. Umweltbildung für Migrant*innen: Die Entwicklung von Screencasts (Erklärvideos). Berlin: IZT - Institut für Zukunftsstudien und Technologiebewertung gemeinnützige $\mathrm{GmbH}$. 
Hartsell, Taralynn, und Steve Chi-Yin Yuen. 2006. «Video streaming in online learning». AACE Journal 14 (1): 31-43.

Hasler, Béatrice Susanne, Bernd Kersten, und John Sweller. 2007. «Learner control, cognitive load and instructional animation». Applied Cognitive Psychology 21 (6): 713-729. https:// doi.org/10.1002/acp.1345.

Heidig, Steffi, Julia Müller, und Maria Reichelt. 2015. «Emotional design in multimedia learning: Differentiation on relevant design features and their effects on emotions and learning». Computers in Human Behavior 44: 81-95. https://doi.org/10.1016/j.chb.2014.11.009.

Hoogerheide, Vincent, Lian Deijkers, Sofie M. M. Loyens, Anita Heijltjes, und Tamara van Gog. 2016. «Gaining from explaining: Learning improves from explaining to fictitious others on video, not from writing to them». Contemporary Educational Psychology 44-45: 95-106. https://doi.org/10.1016/j.cedpsych.2016.02.005.

Hoogerheide, Vincent, Sofie M. M. Loyens, und Tamara van Gog. 2014a. "Comparing the effects of worked examples and modeling examples on learning». Computers in Human Behavior 41: 80-91. https://doi.org/10.1016/j.chb.2014.09.013.

Hoogerheide, Vincent, Sofie M. M. Loyens, und Tamara van Gog. 2014b. «Effects of creating video-based modeling examples on learning and transfer». Learning and Instruction 33: 108-119. https://doi.org/10.1016/j.learninstruc.2014.04.005.

Hoogerheide, Vincent, Sofie M. M. Loyens, und Tamara van Gog. 2016. «Learning from video modeling examples: Does gender matter?» Instructional Science 44 (1): 69-86. https://doi. org/10.1007/s11251-015-9360-y.

Hoogerheide, Vincent, Margot van Wermeskerken, Sofie M. M. Loyens, und Tamara van Gog. 2016. «Learning from video modeling examples: Content kept equal, adults are more effective models than peers». Learning and Instruction 44: 22-30. https://doi.org/10.1016/j. learninstruc.2016.02.004.

Hoogerheide, Vincent, Margot van Wermeskerken, Hilke van Nassau, und Tamara van Gog. 2018. «Model-observer similarity and task-appropriateness in learning from video modeling examples: Do model and student gender affect test performance, self-efficacy, and perceived competence?» Computers in Human Behavior. https://doi.org/10.1016/j.chb.2017.11.012.

Karppinen, Päivi. 2005. «Meaningful learning with digital and online vidoes: Theoretical perspectives». AACE Journal 13 (3): 233-250.

Kizilcec, René F., Jeremy N. Bailenson, und Charles J. Gomez. 2015. «The instructor's face in video instruction: Evidence from two large-scale field studies». Journal of Educational Psychology 107 (3): 724-739. https://doi.org/10.1037/edu0000013.

Knaus, Thomas, und Katrin Valentin. 2016. «Video-Tutorials in der Hochschullehre: Hürden, Widerstände und Potentiale». In Wi(e)derstände, herausgegeben von Thomas Knaus und Olga Engel, 151-181. München: kopaed.

Koning, Björn B. de, Huib K. Tabbers, Remy M. J. P. Rikers, und Fred Paas. 2007. «Attention cueing as a means to enhance learning from an animation». Applied Cognititive Psychology 21 (6): 731-746. https://doi.org/10.1002/acp.1346. 
Krämer, Andreas, und Sandra Böhrs. 2017. «How do consumers evaluate explainer videos? An empirical study on the effectiveness and efficiency of different explainer video formats». Journal of Education and Learning 6 (1): 254-266. https://doi.org/10.5539/jel.v6n1p254.

Leinhardt, Gaea. 2001. «Instructional explanations: A commonplace for teaching and location for contrast». In Handbook of research on teaching, herausgegeben von Virginia Richardson, 333-357. Washington: American Educational Research Association.

Leinhardt, Gaea. 2010. «Introduction: Explaining instructional explanations». In Instructional explanations in the disciplines, herausgegeben von Mary Kay Stein und Linda Kucan, 1-5. New York and Dordrecht and Heidelberg and London: Springer.

Lloyd, Steven A., und Chuck L. Robertson. 2012. "Screencast tutorials enhance student learning of statistics». Teaching of Psychology 39 (1): 67-71. https://doi. org/10.1177/0098628311430640.

Lübcke, Eileen, und Joanna Burchert. 2013. «Berufliches Lernen unter den Bedingungen Digitaler Medien: Videos von Auszubildenden als User Generated Context».bwp@Berufs- und Wirtschaftspädagogik - online, Nr. 24: 1-15. www.bwpat.de/ausgabe24/luebcke_burchert_ bwpat24.pdf.

Lübcke, Eileen, und Joanna Burchert. 2014. «Kongruenz oder Korrespondenz? Soziokulturelle Ökologie als Ansatz zum Verstehen des Web 2.0 in der beruflichen Bildung». In Lernen im Web 2.0, herausgegeben von Nicole C. Krämer, Nicole Sträfling, Nils Malzahn, und Tina Ganster, 207-225. Bielefeld: W. Bertelsmann Verlag.

Mayer, Richard E. 2009. Multimedia learning. 2. Aufl. New York: Cambridge University Press.

Mayer, Richard E., und Paul Chandler. 2001. «When learning is just a click away: Does simple user interaction foster deeper understanding of multimedia messages?» Journal of Educational Psychology 93 (2): 390-397. https://doi.org/10.1037//0022-0663.93.2.390.

Medienpädagogischer Forschungsverbund Südwest. 2017. JIM-Studie: Jugend, Information, (Multi-) Media. https://www.mpfs.de/fileadmin/files/Studien/JIM/2017/JIM_2017.pdf.

Meij, Hans van der, und Jan van der Meij. 2014. «A comparison of paper-based and video tutorials for software learning». Computers \& Education 78: 150-159. https://doi.org/10.1016/j. compedu.2014.06.003.

Merkt, Martin. 2012. «Optimizing the use of videos in education». Dissertation, Tübingen: Eberhard Karls Universität.

Merkt, Martin, und Stephan Schwan. 2014. «How does interactivity in videos affect task performance?» Computers in Human Behavior 31: 172-181. https://doi.org/10.1016/j. chb.2013.10.018.

Merkt, Martin, und Stephan Schwan. 2016. "Lernen mit digitalen Videos». Psychologische Rundschau 67 (2): 94-101. https://doi.org/10.1026/0033-3042/a000301.

Merkt, Martin, Sonja Weigand, Anke Heier, und Stephan Schwan. 2011. «Learning with videos vs. learning with print: The role of interactive features». Learning and Instruction, Nr. 21: 687-704. https://doi.org/10.1016/j.learninstruc.2011.03.004. 
Moreno, Roxana, und Richard E. Mayer. 2007. «Interactive multimodal learning environments». Educational Psychology Review 19 (3): 309-326. https://doi.org/10.1007/s10648-007-90472.

Ouwehand, Kim, Tamara van Gog, und Fred Paas. 2015. «Designing effective video-based modeling examples using gaze and gesture cues». Educational Technology \& Society 18 (4): 78-88.

Pauli, Christine. 2015. «Einen Sachverhalt erklären.» Pädagogik 67 (3): 44-47.

Plass, Jan L., Steffi Heidig, Elizabeth O. Hayward, Bruce D. Homer, und Enjoon Um. 2014. «Emotional design in multimedia learning: Effects of shape and color on affect and learning». Learning and Instruction 29: 128-140. https://doi.org/10.1016/j.learninstruc.2013.02.006.

Rummler, Klaus. 2017. Lernen mit Online-Videos: Eine Einführung. medienimpulse - Beiträge zur Medienpädagogik. https://journals.univie.ac.at/index.php/mp/article/view/mi1041.

Rummler, Klaus, und Karsten D. Wolf. 2012. «Lernen mit geteilten Videos: aktuelle Ergebnisse zur Nutzung, Produktion und Publikation von online-Videos durch Jugendliche». In MEDIEN - WISSEN - BILDUNG, herausgegeben von Wolfgang Sützl, Felix Stalder, Ronald Maier, und Theo Hug, 253-266. Innsbruck: Innsbruck University Press. http://www.uibk.ac.at/ iup/buch_pdfs/9783902811745.pdf.

Schaarschmidt, Nadine, Claudia Albrecht, und Claudia Börner. 2016. «Videoeinsatz in der Lehre: Nutzung und Verbreitung in der Hochschule». In Teaching Trends 2016, herausgegeben von Wolfgang Pfau, Caroline Baetge, Svenja Mareike Bedenlier, Carina Kramer, und Joachim Stöter, 39-49. Münster and New York: Waxmann.

Schnotz, Wolfgang. 2011. Pädagogische Psychologie kompakt. 2., überarb. und erw. Aufl. Weinheim: Beltz.

Schön, Sandra. 2013. «Klappe zu! Film ab! Gute Lernvideos kinderleicht erstellen». In Lernen mit Videos und Spielen, herausgegeben von Jutta Pauschenwein, 3-10. Graz: FH Joanneum.

Schunk, Dale H. 1987. «Peer models and children's behavioral change». Review of Educational Research 57 (2): 149-174. https://doi.org/10.3102/00346543057002149.

Schwan, Stephan, und Roland Riempp. 2004. «The cognitive benefits of interactive videos: Learning to tie nautical knots». Learning and Instruction 14 (3): 293-305. https://doi. org/10.1016/j.learninstruc.2004.06.005.

Shelton, Catharyn C., Annie E. Warren, und Leanna M. Archambault. 2016. «Exploring the use of interactive digital storytelling video: Promoting student engagement and learning in a university hybrid course». TechTrends 60 (5): 465-474. https://doi.org/10.1007/s11528-0160082-z.

Slopinski, Andreas. 2016. «Selbstbestimmt motiviertes Lernen durch die Produktion von Lernund Erklärvideos». Medienproduktion: Online-Zeitschrift für Wissenschaft und Praxis 10 (2016): 9-13. http://www5.tu-ilmenau.de/zeitschrift-medienproduktion/wordpress/wpcontent/A10/3_Slopinski.pdf. 
Spanjers, Ingrid A. E., Tamara van Gog, Pieter Wouters, und Jeroen J. G. van Merriënboer. 2012. «Explaining the segmentation effect in learning from animations: The role of pausing and temporal cueing». Computers \& Education 59 (2): 274-280. https://doi.org/10.1016/j.compedu.2011.12.024.

Spires, Hiller A., Lisa G. Hervey, Gwynn Morris, und Catherine Stelpflug. 2012. «Energizing project-based inquiry: Middle-grade students read, write, and create videos». Journal of Adolescent \& Adult Literacy 55 (6): 483-493. https://doi.org/10.1002/JAAL.00058.

Wermeskerken, Margot van, und Tamara van Gog. 2017. «Seeing the instructor's face and gaze in demonstration video examples affects attention allocation but not learning». Computers \& Education 113: 98-107. https://doi.org/10.1016/j.compedu.2017.05.013.

Wermeskerken, Margot van, Bianca Grimmius, und Tamara van Gog. 2018. «Attention to the model's face when learning from video modeling examples in adolescents with and without autism spectrum disorder». Journal of Computer Assisted Learning 34 (1): 32-41. https://doi.org/10.1111/jcal.12211.

Wermeskerken, Margot van, Susanna Ravensbergen, und Tamara van Gog. 2017. «Effects of instructor presence in video modeling examples on attention and learning». Computers in Human Behavior. https://doi.org/10.1016/j.chb.2017.11.038.

Wittwer, Jörg, und Alexander Renkl. 2008. «Why instructional explanations often do not work: A framework for understanding the effectiveness of instructional explanations». Educational Psychologist 43 (1): 49-64. https://doi.org/10.1080/00461520701756420.

Wolf, Karsten D. 2015. «Video-Tutorials und Erklärvideos als Gegenstand, Methode und Ziel der Medien- und Filmbildung». In Filmbildung im Wandel, herausgegeben von Anja HartungGriemberg, Thomas Ballhausen, Christine Trültzsch-Wijnen, Alessandro Barberi, und Katharina Kaiser-Müller, 121-131. Wien: new academic press. http://www.medienimpulse.at/ files/get/6d728c2ec512a214ed7891463e9b37c7/II\%20Reihe\%20MI\%202015\%20Filmbildung.pdf.

Zahn, Carmen, Beatriz Barquero, und Stephan Schwan. 2004. «Learning with hyperlinked videos: Design criteria and efficient strategies for using audiovisual hypermedia». Learning and Instruction 14 (3): 275-291. https://doi.org/10.1016/j.learninstruc.2004.06.004.

Zahn, Carmen, Karsten Krauskopf, Friedrich W. Hesse, und Roy Pea. 2010. «Digital video tools in the classroom: Empirical studies on constructivist learning with audio-visual media in the domain of history». In Learning in the disciplines, herausgegeben von Kimberley Gomez, Leilah Lyons, und Joshua Radinsky, 620-627. Chicago: IL: Society of the Learning Sciences.

Zhang, Dongsong, Lina Zhou, Robert O. Briggs, und Jay F. Nunamaker. 2006. «Instructional video in e-learning: Assessing the impact of interactive video on learning effectiveness». Information \& Management 43 (1): 15-27. https://doi.org/10.1016/j.im.2005.01.004. 